\title{
Magnetic Measurement of Quadrupole and Sextupole Magnets for the MIT-Bates South Hall Ring (SHR)
}

\author{
D.R. Tieger, J.D. Zumbro* and W.W. Sapp \\ Bates Linear Accelerator Center \\ Middleton, MA 01949
}

\section{Abstract}

Final results of magnetic measurements of 128 quadrupoles and 32 sextupoles are presented. There are many places in the SHR complex where these magnets will be operated from a common power supply. For the quadrupole magnets there are 4 octets, 4 triplets and 18 doublets; these magnets have been matched to $\pm 0.1 \%$ in excitation response for the operating range corresponding to $0.3-1.0 \mathrm{GeV}$. For the sexlupoles there are four octets where the (pairwise) matching has generally been made to the $\pm 0.3 \%$ level. Parameterization of the data as well as fits are described and examples of the magnet groupings are shown.

\section{INTRODUCTION}

The $190 \mathrm{~m}$ MIT-Bates South Hall Ring (SHR) will provide both high $(85 \%)$ duty-factor extracted beams as well as high current (up to $80 \mathrm{~mA}$ ) circulating beams for internal target physics in the energy range of $300 \mathrm{MeV}$ to $1 \mathrm{GeV}$. The project has been described elsewhere $\mathrm{e}^{[1]}$. In order to complete the SHR project, a total of 128 quadrupoles and 32 sextupoles were manufactured. Magnetic measurements have been made on each of these multipole elements in order to determine harmonic content and similarity of response, since many of these magnets will be powered from common power supplies. Previously, we have described these quadrupoles and shown results from the first 24 of them to be measured ${ }^{[2]}$. In that reference we described the magnetic measurement technique and the equipment that was used, thus we shall only summarize this information here. The sextupoles are replicas of LBL Light Source-Booster sextupoles ${ }^{[3]}$, which were fabricated for us by Everson Electric ${ }^{[4]}$.

\section{MEASUREMENTS AND RESULTS}

Our multipole magnetic measurement equipment consists of a harmonic analyzer table which was built for us at Chalk River ${ }^{[3]}$. The heart of this system is a ceramic bobbin that contains two tangential coils (one with $90^{\circ}$ and one with $45^{\circ}$ nominal opening angle) which is rotated on the axis of the multipole. The signal from one of the coils is input to a voltage-to-frequency ( $\mathrm{V} / \mathrm{f})$ converter with $1 \mathrm{MHz}$ per full scale bipolar output. Pulses from the $\mathrm{V} / \mathrm{f}$ are accumulated in 256 bins per each revolution of the bobbin. Data are accumulated with a PC, which is also used to cycle the power supply controlling the multipole. At each current setting a total of 10 data sets is obtained, 5 with the bobbin rotating in one direction and 5 with the rotation in the other direction. Prior to a set of measurements, the bobbin is centered on the magnetic axis by minimizing the dipole (quadrupole) term for the quadrupole (sextupole) magnet to be measured, using the $90^{\circ}\left(45^{\circ}\right)$ coil.
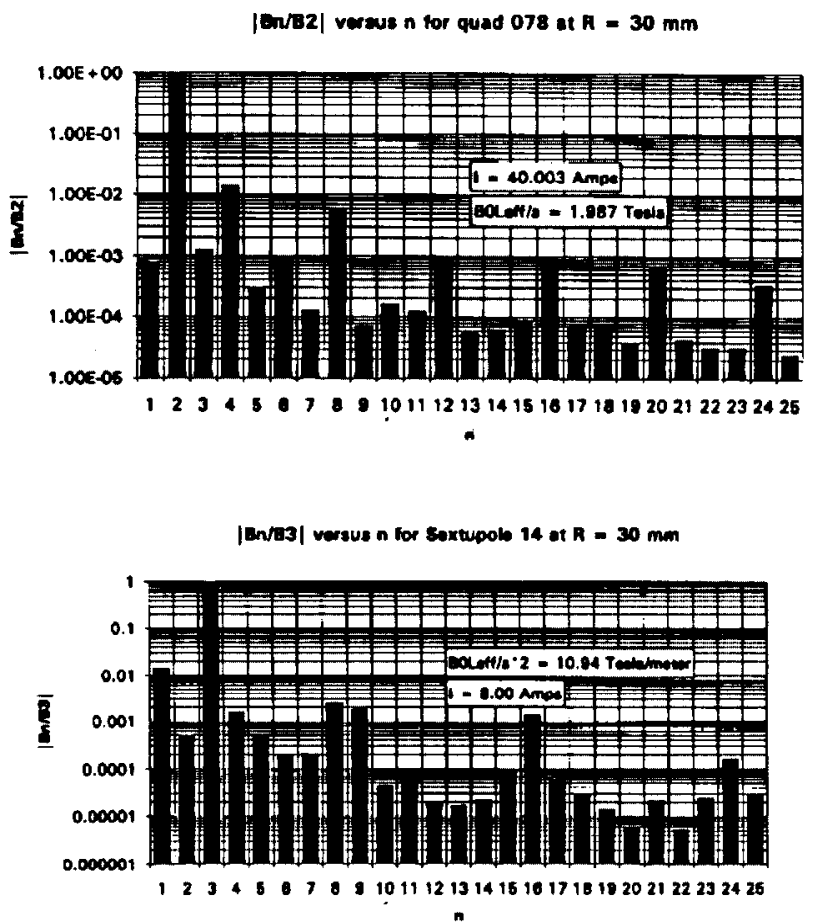

Figure 1. The relative harmonic strengths for a) typical quad at 40 Amps $\left(90^{\circ}\right.$ coil not sensitive to $\mathrm{n}=4,8,12$,etc. $)$ and b) typical sextupole at $8 \mathrm{Amps}\left(45^{\circ}\right.$ coil not sensitive to $n=8,16,24$,etc.)

The data are then analyzed with a Fast-Fourier Transform (FFT) from which the harmonic strengths are obtained. Figure 1 shows the harmonics relative to the desired one for both a typical quadrupole and sextupole, where in both cases the correct magnet and coil geometry is used. For the quadrupoles the quantity $\mathrm{B}_{0} \mathrm{~L}_{\text {eff }} / \mathrm{a}(\mathrm{I})$ is obtained, while for the sextupoles the quantity obtained is $B_{0} L_{e f f} / a^{2}(I)$, where in both cases the correct magnet and coil geometry is used. For our quads, the effective length is $300 \mathrm{~mm}$ and the aperture radius is $32.5 \mathrm{~mm}$, while for the sextupoles, the effective length is $121 \mathrm{~mm}$ and

* This work is supported in part by the U.S. Department of Energy under contract \# DE-AC02-76ER03069. 

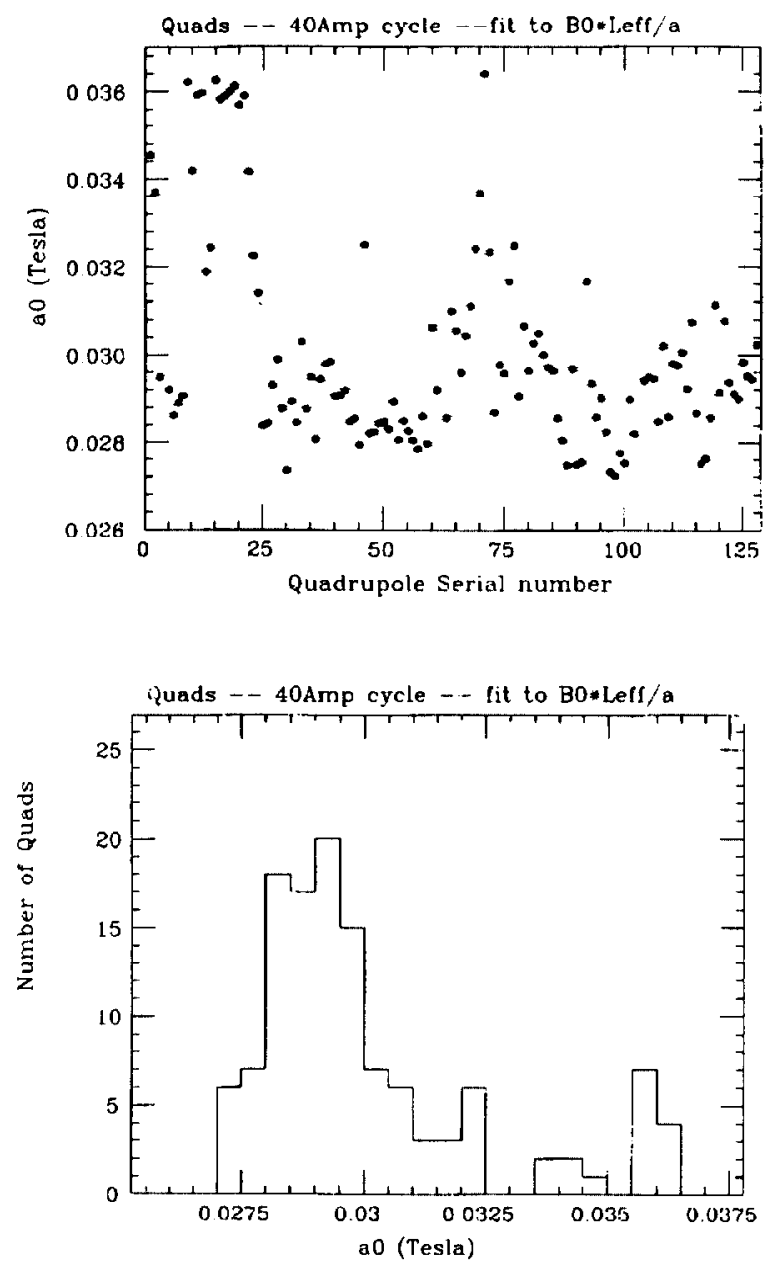

Figure 2. The residual field terms $\mathrm{a}_{0}$ obtained from fits to Eq. 1 for the $40 \mathrm{Amp}$ cycle data. a) shows the scatter vs. Serial Number and b) shows the statistics of the distribution.

aperture radius is $35 \mathrm{~mm}$. We then fit the obtained strengths to the form (e.g. for the quads):

$$
\begin{gathered}
B_{0} L_{\text {eff }} / a(I)=a_{0}+a_{1}(I / 150)+a_{5}(I / 150)^{5}+ \\
a_{9}(I / 150)^{9}+a_{13}(I / 150)^{13}
\end{gathered}
$$

from which we can make comparisons and look for matches. Initially, we measured each quad on each of 5 different cycle currents (corresponding to the maximum values of the quad power supplies), which proved to be very time intensive. After approximately two-thirds of them had been measured and we were beginning to make tentative matches, we realized that about one-third of them would require polarity changing. At that point we decided to measure each quad on only one common cycle and make choices based upon that cycle's results. Our SHR lattice, which contains 79 quadrupoles, has four octets-all 32 of these quads are on 40 Amp cycles, which therefore was the cycle of choice. The remaining quadrupoles were all scanned at $40 \mathrm{Amps}$. Shown in Figure 2 are the residual field terms $a_{0}$ obtained from fits of all our quadrupoles to Eq. 1 for the $40 \mathrm{Amp}$ cycle data. It should be pointed out that with our $\mathrm{L}_{\text {eff }} / \mathrm{a}$, the residual fields are scattered about a value of roughly 30 Gauss. Shown in Figure 3 are the linear terms $a_{1}$ in the fit to the data, and one can see that there is a natural
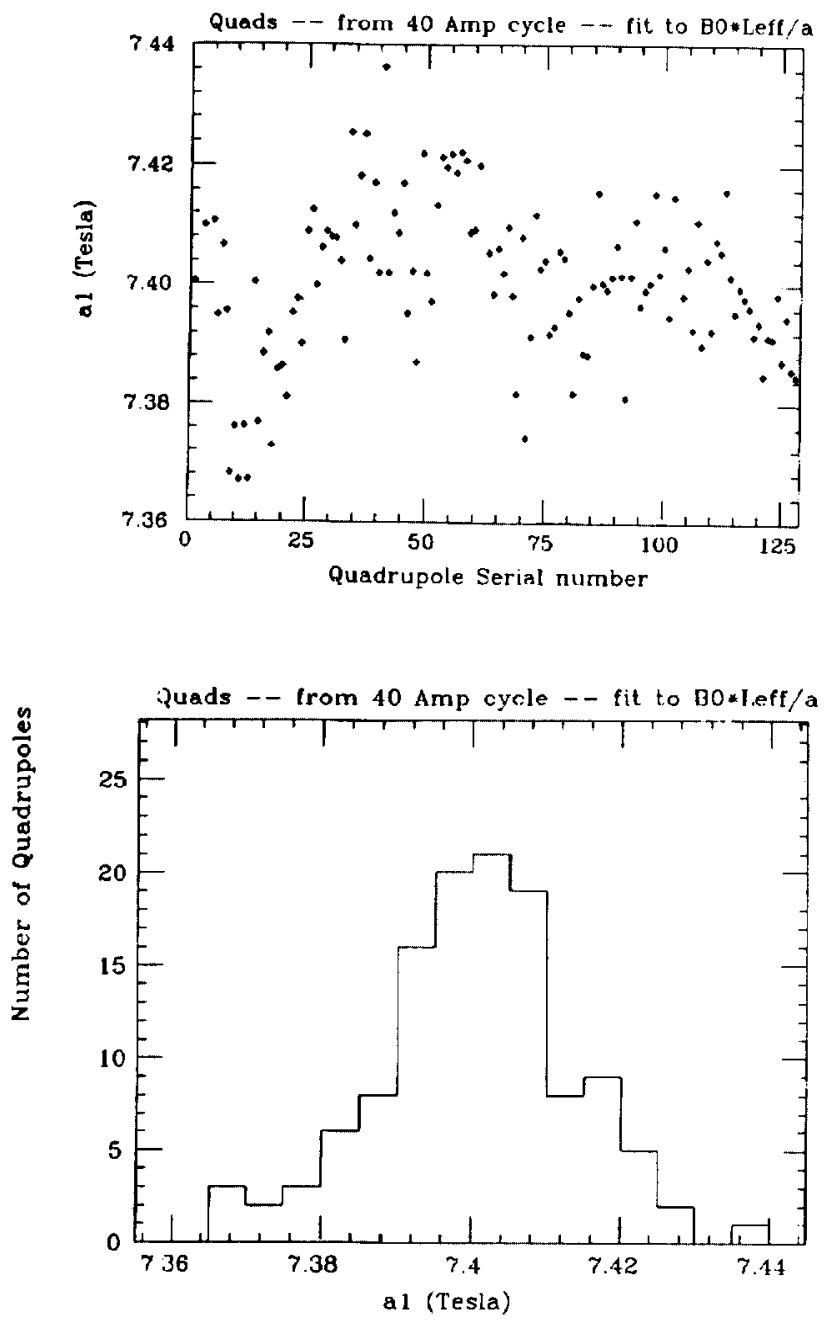

Figure 3. Similar to Figure 2 for the linear term $a_{1}$ from fits to the data for the 40 Amp cycle data.

spread of $\pm 5 \%$ in the distribution, which is much narrower than the spread in the residual field term. However, the criterion that the accelerator physicists had given us was to match quads to $\pm .1 \%$, over the operating range of the SHR. We were able to meet this criterion and two examples are shown in Figure 4. Each arc of the lattice has a triplet ( $60 \mathrm{Amp}$ positive polarity) in addition to an octet (40 Amp negative polarity). The data in Figure 4 are shown relative to the fit for the reference quad, and one can see that the fit meets the criterion over the range of $8 \mathrm{Amps}$ to $25 \mathrm{Amps}$, which is the operational range of the octet.

For the sextupoles, all magnets were measured on a single $8 \mathrm{Amp}$ cycle. Half were chosen to be one polarity (deflecting electrons to the right) and half the other. We then pair-wise matched them to approximately $\pm .3 \%$ over the operating range of the sextupoles, which was deemed acceptable to the accelerator physicists.

An example of the sextupole matching is shown in Figure 5 .

In conclusion, we have met the design criteria of the SHR in the placement of the quadrupole and sextupole multipole elements. From the 128 quadrupoles it was just possible to select the octets, within each of which the 

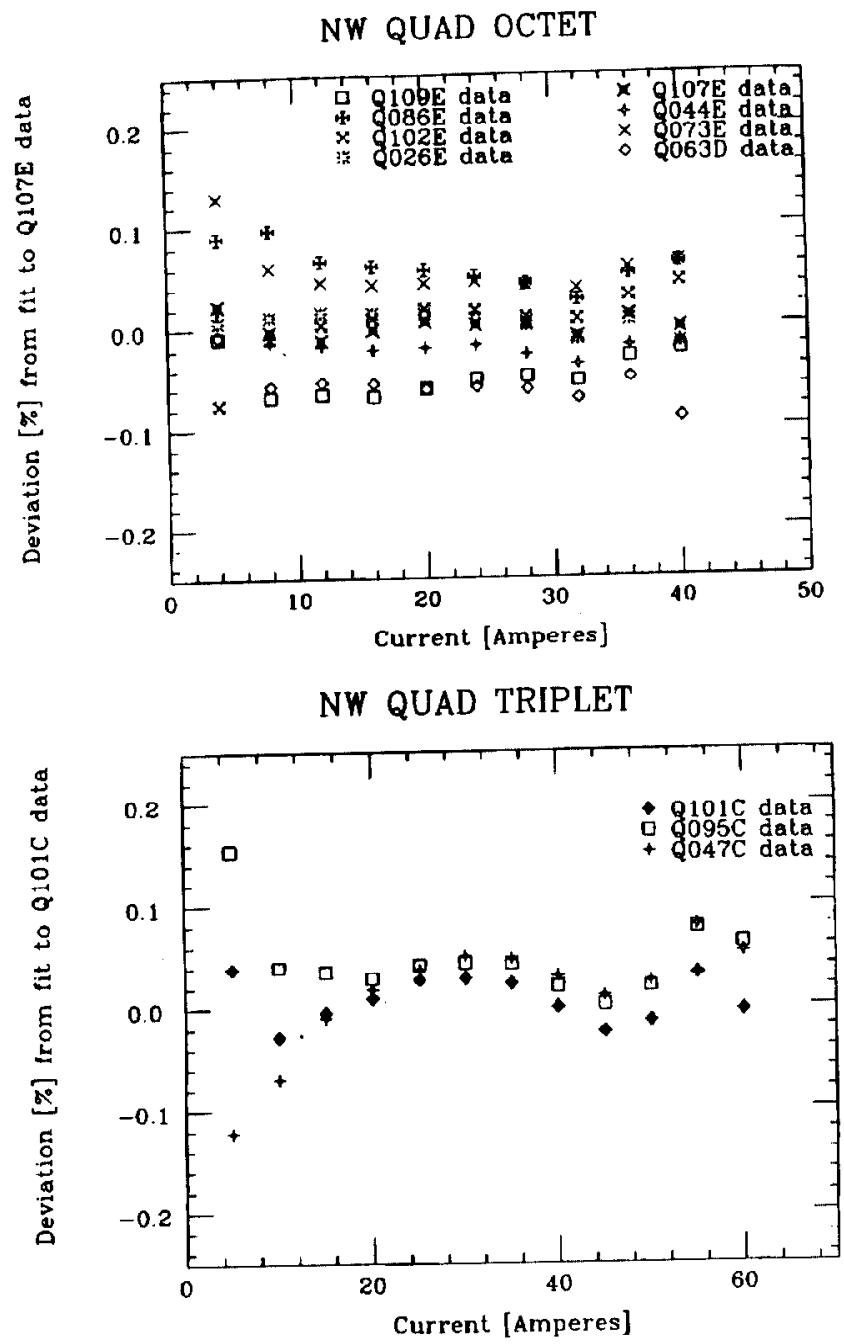

Figure 4. a) Shows the data for the NW Arc octet relative to the fit to the reference quad data. b) shows the data for the triplet in the same arc.

response was matched to $\pm 0.1 \%$. This was the most stringent requirement. The sextupoles showed a somewhat larger variation in their strengths, but it was still possible to obtain pairs matched to $\pm 0.3 \%$. The fabrication of both types of multipoles followed standard practices, and there was no post-fabrication machining or selection of materials before final assembly. For applications demanding better matching (than has been demonstrated here), improved fabrication techniques and tighter quality assurance will be necessary.

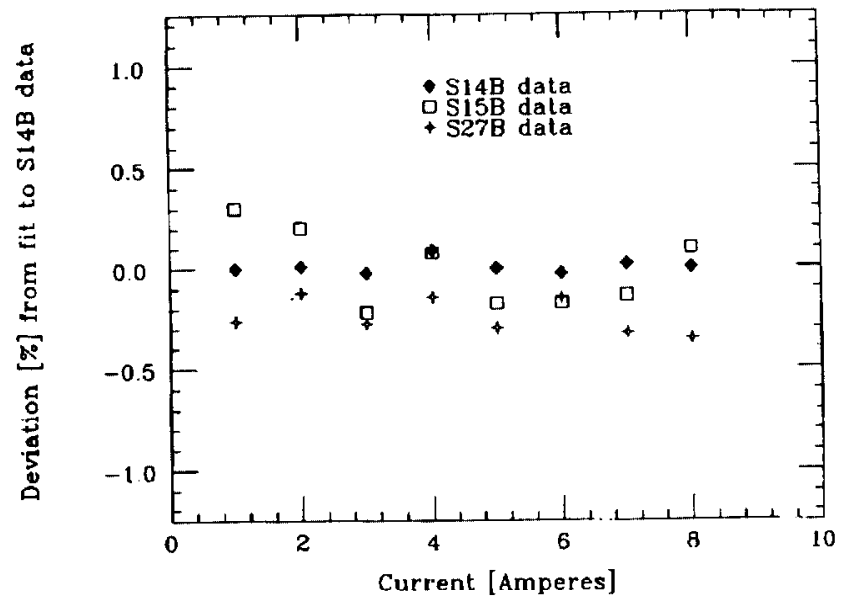

Figure 5. Shows the data from three sextupoles compared to the fit for one of them.

\section{REFERENCES}

[1] J.B. Flanz et al., Proceedings of the 1989 IEEE Particle Accelerator Conf. March 20-23, 1989, p.34.

[2] J.D. Zumbro et al., Proceedings of the 1991 IEEE Particlc Accelerator Conf., May 6-9, 1991.

[3] J. Tanabe, S. Marks, K. Luchini, Lawrence Berkeley Laboratory, Engineering Note M6816, 1989.

[4] Everson Electric Company, Lehigh, PA

[5] W.G. Davies, N. Bray and R. Howard, Chalk River Nuclear Laboratories, Chalk River, Ont., Canada

* Present Address: LAMPF, Los Alamos National Lab, Los Alamos, NM 87545 